\title{
Surface plasmon resonance sensors based on uniform-waist tapered fibers in a reflective configuration
}

\author{
Óscar Esteban, Natalia Díaz-Herrera, María-Cruz Navarrete, and Agustín González-Cano
}

\begin{abstract}
We present a configuration for surface plasmon resonance sensors based on uniform-waist tapered optical fibers and reflective elements. Once the fiber is tapered fulfilling the adiabatic criterion, a multilayer including a metallic medium is asymmetrically deposited on the uniform waist of the fiber. This feature provides the resonant excitation of multiple surface plasma waves. In addition, a mirror is produced at the fiber tip by a chemical Tollens reaction. In this way, the sensor operates in a reflective mode, more convenient for dip probes. When these sensors are spectrally interrogated, a high sensitivity of $10^{-4}$ refractive index units per nanometer is attained. These devices can be advantageously used for any kind of chemical sensing and biosensing. () 2006 Optical Society of America

OCIS codes: $240.6680,060.2370$.
\end{abstract}

\section{Introduction}

During the past few years, the use of surface plasmon resonance- (SPR) based sensors remarkably increased in the fields of chemical and biochemical sensing. ${ }^{1}$ The most extended arrangement is based on the Kretschmann configuration. Several interrogation methods can be used with this setup. The most widely used is the angular one, ${ }^{1-3}$ although reflectance, SPR wavelength $\left(\lambda_{\mathrm{SPR}}\right)$ tracking, phase shift, and even polarization measures have also been reported. ${ }^{1}$ However, fiber-optic sensors can provide simpler, smaller, and more compact setups when an on-line measurement is needed. In this context, a number of fiber-optic sensors based on SPR have proved to be useful for chemical and biochemical sensing either for remote or in situ measurements. These sensors are mainly based on the interaction between the evanescent field of the guided radiation inside the optical fiber and a transducer including a

O. Esteban (oscar@depeca.uah.es) is with the Departamento de Electrónica, Universidad de Alcalá, Escuela Politécnica, Alcalá de Henares, Madrid 28871, Spain. N. Díaz-Herrera and A. GonzálezCano are with the Departamento de Óptica, Escuela Universitaria de Óptica, Universidad Complutense de Madrid, Arcos de Jalón, s/n, Madrid 28037, Spain. M.-C. Navarrete is with the Departamento de Óptica, Facultad de CC. Físicas, Universidad Complutense de Madrid, Ciudad Universitaria, s/n, Madrid 28040, Spain.

Received 21 February 2006; revised 21 April 2006; accepted 26 April 2006; posted 28 April 2006 (Doc. ID 68274).

$0003-6935 / 06 / 287294-05 \$ 15.00 / 0$

(C) 2006 Optical Society of America metallic medium, which must be functionalized when applied to a specific molecular detection. ${ }^{1}$ To reach the evanescent field, a number of techniques have been employed. Side-polished fibers (D-type fibers) have been widely used. ${ }^{4-6}$ Another option is chemical etching with hydrofluoric acid (HF) to eliminate fiber cladding. ${ }^{7,8}$ The mechanical strip of the fiber cladding has also been used. ${ }^{8}$ However, these methods only provide access to a relatively small intensity of the evanescent field, and some of these options increase the fragility of the fiber. Recently, tapered fiber have shown their potential since the evanescent field intensity is higher than in the previous configurations. Two main types of tapered fiber have been reported. The first one is the so-called biconical taper, ${ }^{9}$ which is usually produced by using a fiber splicer and provides short and sharp devices with high losses. The second one is the so-called adiabatic taper, which is much longer than the biconical ones. These devices are produced with the traveling-burner technique, ${ }^{10-12}$ where the fiber is gently stretched while heated. Details are given elsewhere. ${ }^{10}$ With this method, it is possible to obtain uniform-waist tapered (UWT) fibers with a controlled length and a waist diameter in a highly reproducible way. Furthermore, SPR sensors with UWT fibers have shown a high sensitivity and dynamic range $\mathrm{e}^{10,13-15}$ and could be the best option when the sample volume is not a critical parameter.

The optical fiber sensors based on evanescent waves and SPR have been used mainly in the transmission mode. This configuration requires a length of fiber after the transducer to return to the control 


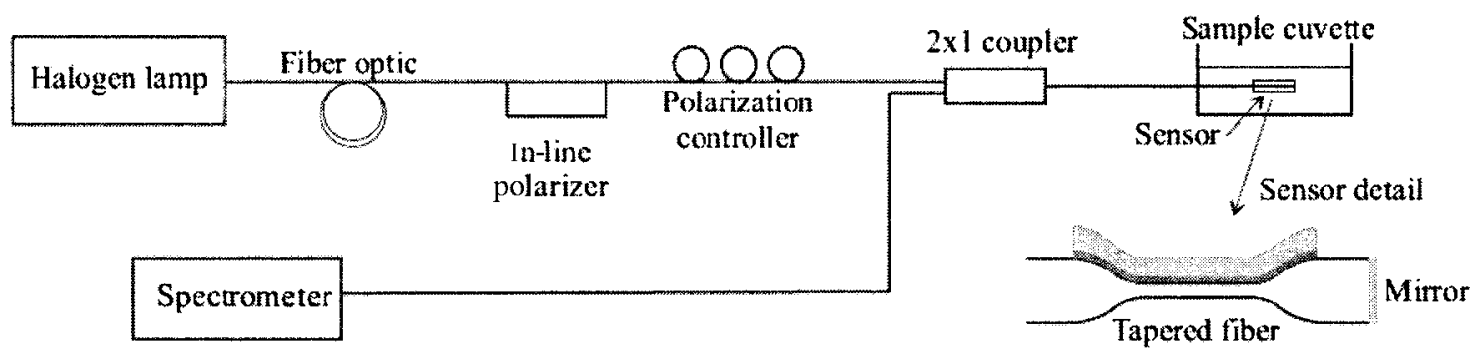

Fig. 1. Schematic of the setup used to measure the spectral sensor response. Side and cross-sectional views of the sensor are also shown.

unit in a loop. For overcoming this feature, some retroreflective SPR devices have been proposed, some of them based on geometric considerations when tapering the fiber ${ }^{16,17}$ and others based on reflective elements added after the transducer. ${ }^{8}$ These devices require complex apparatuses and techniques for their construction when a reproducible batch of sensors is to be produced, their main advantage being the small size, which makes them suitable for integration in microwell plates ${ }^{16}$ and in vivo measurements. ${ }^{17}$

However, the small size of SPR sensors can also negatively affect their sensitivity, since in tapered fiber-based SPR sensors, this sensitivity is directly related to the interaction length.

Here we present and characterize a configuration for SPR sensors based on UWT fibers with a reflective element added at the fiber tip after the transducer. The reflective element is a $\mathrm{Ag}$ mirror deposited at the fiber end by chemical means following the Tollens reaction, which is an easy and cheap method to obtain reflective devices. ${ }^{18}$ As a transducer, a double asymmetrical layer has been used, and a remarkable feature of the sensor response is its relative immunity to the polarization of the incident light. As we will show, the sensitivity of these devices is very good and they are good candidates for any kind of chemical sensing or biosensing.

\section{Experimental Arrangement}

The sensors' fabrication procedure involves three steps that have been depicted elsewhere. ${ }^{10,13}$ The first one is decreasing the fiber diameter so that we can reach the evanescent field of the mode propagated by the unperturbed fiber. We follow the procedure developed by Kenny et al., ${ }^{12}$ the so-called traveling burner. As mentioned above, with this procedure the fiber is gently stretched while it is being heated, so core and cladding diameters eventually decrease up to core collapse. The obtained devices show low losses, which is an important feature for distributed and remote sensing. This technique has been widely used by different authors ${ }^{9,10,13-15}$ to make a great variety of devices based on tapered optical fibers. With this procedure, we can obtain different substrates with different uniform waists of tapered fiber over a length of several millimeters with low loss. The optical fiber that we use is a conventional single-mode step-index fiber at a nominal wavelength of $820 \mathrm{~nm}$. The waist of the tested devices is $35 \mu \mathrm{m}$, its length is about $6 \mathrm{~mm}$, and the losses of the tapers are less than $0.3 \mathrm{~dB}$.

The second step of the production process consists of the deposition of layers on the substrates obtained in the first step explained above. The deposition method is physical vapor deposition, as depicted by Alonso et al. ${ }^{5}$ The asymmetric device is thus obtained by deposition of a double layer on one side of the tapered fiber.

The values of the thickness of materials deposited vary from zero at the edges to a maximum value at the top of the waist. We have chosen a first layer of $\mathrm{Al}$ $8 \mathrm{~nm}$ thick and a second layer of $\mathrm{TiO}_{2} 60 \mathrm{~nm}$ thick. The $\mathrm{Al}$ shows better adherence than $\mathrm{Au}$ to $\mathrm{SiO}_{2}$ fibers, and it is best suited for SPR at wavelengths in the $600-1000 \mathrm{~nm}$ range, while the $\mathrm{TiO}_{2}$ layer is used to tune the SPR response to the refractive index range of interest, ${ }^{13,14}$ which relies on the interval $1.32-1.40$ of aqueous solutions at wavelengths in the visiblenear-infrared spectral range.

Finally, the fiber, after the transducer is cleaved, is immersed in a vessel where a Tollens reaction takes place. ${ }^{18}$ Then, a thick layer of $\mathrm{Ag}$ is deposited at the fiber tip, providing reflectance up to $90 \%-95 \%$ in a few minutes. The fiber end is further encapsulated with an epoxy resin to prevent $\mathrm{Ag}$ oxidation. A schematic of the complete device is shown in Fig. 1.

The constructed sensors have been spectrally interrogated to determine their behavior against refractive index (RI) changes of the medium surrounding them. The experimental setup can be seen in Fig. 1, where a halogen lamp (broadband spectrum) is the light source. Because of the asymmetry of the structure, a dependence on the polarization of the propagated modes inside the UWT is expected, since SPR happens only when the electrical field of the propagated mode is normal to the interface between metal and dielectric media. Thus an in-line polarizer $^{19}$ has been added and a set of Lèfevre loops is used to control the polarization plane of the resultant linearly polarized light. With this polarization stage, we can optimize the contrast between dips in the reflected spectrum without strictly choosing either TM or TE polarization. Then a $2 \times 1$ fiber coupler takes the optical radiation to the transducer. The light passes through this one, is reflected back at the fiber tip, and passes again through the transducer toward the coupler where it is brought to a CCD spectrograph. There is a double interaction 


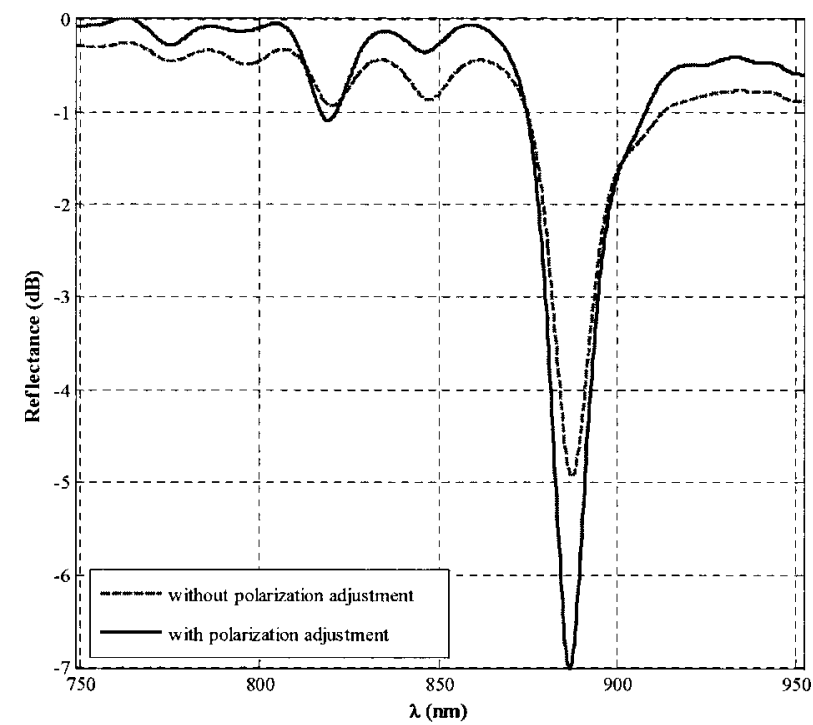

Fig. 2. Spectral response when the outer medium is pure water. Dashed curve represents the sensor response for the unoptimized polarization of the input light. Optimization of the polarization direction provides the response represented on the solid curve.

with the transducer, which increases the intensity of the resonance making the device analog to a doublesided deposited taper. ${ }^{15}$

\section{Experimental Results}

The tests performed are based on the changes of the spectral transmittance of the device with the variations of the outer medium RI, using the transmittance when the surrounding medium is air as a reference. At first, the dependence of the response with the polarization of incident modes was checked for pure water as the outer medium. The results can be seen in Fig. 2. The response obtained for the polarized input light without a polarization plane adjustment is plotted on the dashed curve, and the solid curve shows the response when the polarizationcontrolling elements are used to maximize the coupling, thus producing deeper dips.

Then, a given concentration of ethylene glycol was added to pure water. The RI of the mixture depends on the ethylene glycol concentration according to the following empirical law ${ }^{13}$ :

$$
n(T)=n_{\mathrm{H}_{2} \mathrm{O}}(T)+0.111 \frac{V_{\text {eth }}}{V_{\text {tot }}},
$$

where $n$ and $n_{\mathrm{H}_{2} \mathrm{O}}$ are the RIs of the solution and pure water, respectively, $V_{\text {eth }}$ is the volume of ethylene glycol, and $V_{\text {tot }}$ is the total volume of the mixture.

With the polarization optimized, we can track the two principal minima with the variation of the RI of the outer medium. The obtained results are shown in Fig. 3, with open and filled circles, respectively, denoting the main and the secondary minima displacement with outer medium RI. The sensitivity is approximately $10^{-4} \mathrm{RI}$ units for a spectral resolution of $1 \mathrm{~nm}$, and the response is linear with the RI, as

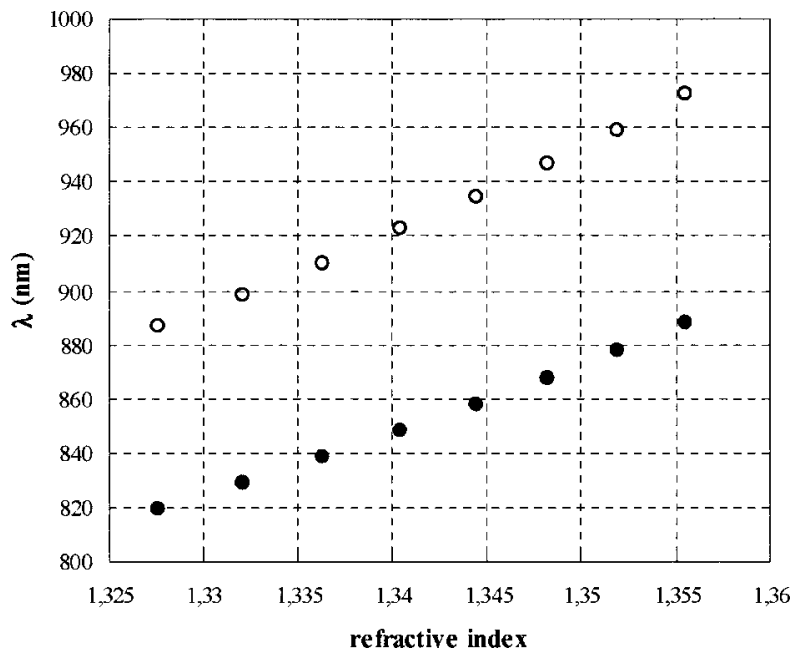

Fig. 3. Displacement of the two main $\lambda_{\mathrm{SPR}}$ shown on the solid curve in Fig. 2 with the outer medium RI changes. Open circles correspond to the main dip and solid circles correspond to the most important secondary dip.

expected for this kind of device. This sensitivity is as high as the ones reported to date. ${ }^{20}$

An additional test was performed with unpolarized input light and no polarization control. The response, in the case of pure water, can be seen in Fig. 4 (solid curve) in comparison with the optimized response obtained in the previous test (dashed curve). It is a remarkable feature that there is only a small redshift in SPR corresponding to a fundamental mode, while resonance intensity decreases slightly together with a broadening of the minimum, but the SPR due to the second-order mode keeps the $\lambda_{\mathrm{SPR}}$. As can be observed, in this case no other dips appear. As in the previous case, we can track the minima with RI changes ob-

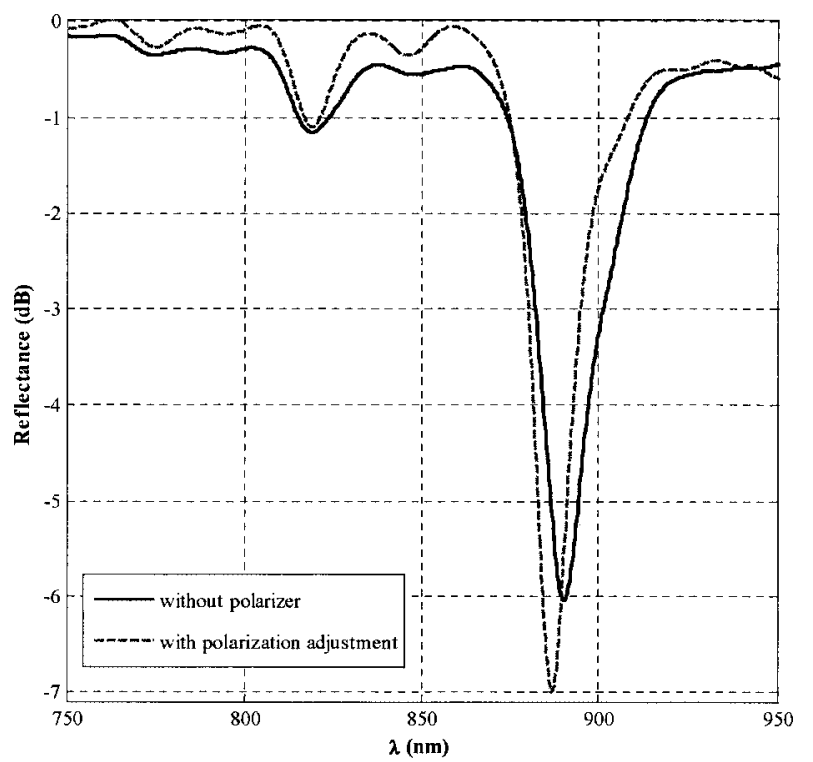

Fig. 4. Spectral response when the outer medium is pure water. The dashed curve represents the optimized polarization response as in Fig. 2. The solid curve is the response for the unpolarized light. 


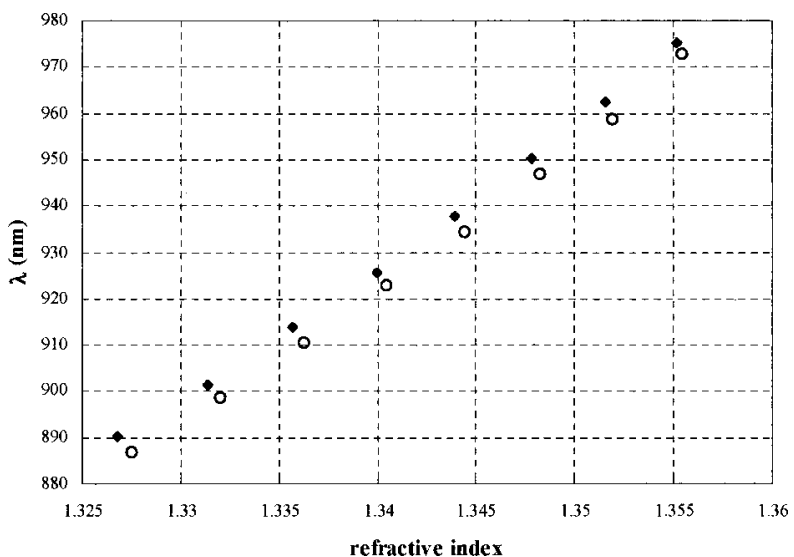

Fig. 5. Comparison between the displacement with the outer medium RI of the main dip of Fig. 4 for the optimized polarization direction (open circles) and unpolarized light (solid diamonds).

taining the same sensitivity. In Fig. 5, the comparison between the operation with optimized polarization (open circles) and without a polarizer (solid diamonds) is shown. This means that we can use these devices virtually as polarization-independent ones. If we are using unpolarized sources, as in our case, the difference between using or not using polarizationcontrolling elements is simply a small constant offset that does not compromise the sensitivity of the device. This feature is important, since we do not need any complex setup to control the polarization orientation of the input light, which implies that the sensor size can be reduced, providing an easy integration in remote or in situ measurement systems.

\section{Conclusions}

A novel configuration for SPR sensors with UWT fibers has been proposed. They are based on tapered fibers with a double-layer deposition and a reflective element at the fiber tip. The sensitivity is the same for transmissive sensors. The employed method to make the mirror at the fiber end is inexpensive and easy to develop since only a chemical reaction must be accomplished. The fabrication of tapered fibers following the traveling-burner technique is a wellknown method, which provides substrates in a very repeatable way. Furthermore, the obtained tapers are more robust than devices based on the same principle obtained by chemical or mechanical methods and provide a higher evanescent field suitable to interact with the deposited transducer and the outer medium.

The deposited layers are optimized to obtain a good response in the visible-near-infrared spectral range for RIs of the outer medium lying in the range of $1.32-1.40$, which is the operating range for most biological measurements. Furthermore, the $\mathrm{TiO}_{2}$ can be functionalized as easily as the Au layer commonly used to date in chemical and biochemical sensors. Since the response is virtually polarization independent, these devices are very small and compact, well suited for integration with compact fluidic systems when the sample volume is not critical. They are also good candidates for dip probes and for the design and production of new sensors.

The authors thank Pieter Swart (Rand Afrikaans University, Johannesburg), Lourdes Sobrino, and Olga Cilleros (Universidad Complutense de Madrid, Spain) for their kind help with the mirror deposition technique. We also thank C. Cosculluela (Universidad de Zaragoza, Spain) for helping us with the production of the transducers.

This work has been partially supported by Comunidad de Madrid (Spain) projects SELENE (Sensores de fibra óptica reflectivos con tecnologia de campo evanescente), ref. GR/MAT/0620/2004, and ROMA (Nueva generación de Refractómetros de fibra Óptica para aplicaciones MedioAmbientales), ref. GR/AMB/ 0615/2004, and by Spanish project NESTOR (Nuevas tecnologias de sensores de fibra óptica para la observación del medio marino), Programa Nacional de Recursos Naturales, Ministerio de Ciencía y Tecnología, ref. CTM2004-03899.

\section{References}

1. J. Homola, S. S. Yee, and G. Gauglitz, "Surface plasmon resonance sensors: review," Sens. Actuators B 54, 3-15 (1999).

2. H. Kano and S. Kawata, "Surface-plasmon sensor absorptionsensitivity enhancement," Appl. Opt. 33, 5166-5170 (1994).

3. G. Orellana, ed., Book of Abstracts, EUROPT(R)ODE VII, (Madrid, Spain 2004).

4. S.-M. Tseng and C.-L. Chen, "Side-polished fibers," Appl. Opt. 31, 3438-3447 (1992).

5. R. Alonso, F. Villuendas, J. Tornos, and J. Pelayo, "New 'inline' optical-fibre sensor based on surface plasmon excitation," Sens. Actuators A 37-38, 187-192 (1993).

6. Ó. Esteban, M. C. Navarrete, A. González-Cano, and E. Bernabeu, "Measurement of the degree of salinity of water with a fiber-optic sensor," Appl. Opt. 38, 5267-5271 (1999).

7. F. J. Liao and J. T. Boyd, "Single-mode fiber coupler," Appl. Opt. 20, 2731-2734 (1981).

8. L. A. Obando, D. J. Gentleman, J. R. Holloway, and K. S. Booksh, "Manufacture of robust surface plasmon resonance fiber optic based dip-probes," Sens. Actuators B 100, 439-449 (2004).

9. C. Fernández-Valdivileso, E. Egozkue, I. R. Matías, F. J. Arregui, and C. Bariáin, "Experimental study of a thermochromic materila based optical fibre sensor for monitoring the temperatura of the water in several applications," Sens. Actuators B 91, 231-240 (2003).

10. J. Villatoro, D. Monzón-Hernández, and E. Mejía, "Fabrication and modeling of uniform-waist single-mode tapered optical fiber sensors," Appl. Opt. 42, 2278-2283 (2003).

11. A. Díez, M. V. Andrés, and J. L. Cruz, "Hybrid surface plasma modes in circular metal-coated tapered fibers," J. Opt. Soc. Am. A 16, 2978-2982 (1999).

12. R. P. Kenny, T. A. Birks, and K. P. Oakey, "Control of optical fiber taper shape," Electron. Lett. 37, 1654-1656 (1991).

13. F. J. Bueno, Ó. Esteban, N. Díaz-Herrera, M. C. Navarrete, and A. González-Cano, "Sensing properties of asymmetric doublelayer covered tapered fibers," Appl. Opt. 43, 1615-1620 (2004).

14. A. González-Cano, F. J. Bueno, Ó. Esteban, N. Díaz-Herrera, and M. C. Navarrete, "Multiple surface plasmon resonante in uniform-waist tapered optical fibers with an asymmetric double-layer deposition," Appl. Opt. 44, 519-526 (2005).

15. D. Monzón-Hernández and J. Villatoro, "High-resolution re- 
fractive index sensing by means of a multiple peak surface plasmon resonance optical fiber sensor," Sens. Actuators B 115, 227-231 (2006).

16. R. C. Jorgenson, "A surface plasmon resonance side active retroreflecting sensor," Sens. Actuators B 73, 236-248 (2001).

17. B. Grunwald and G. Holst, "Fibre optic refractive index microsensor based on white-light SPR excitation," Sens. Actuators A 113, 174-180 (2004).

18. Y. Saito, J. J. Wang, D. A. Smith, and D. N. Batchelder, "A simple chemical method for the preparation of silver surfaces for efficient SERS," Langmuir 18, 2959-2961 (2002).

19. R. Alonso, J. Subias, J. Pelayo, F. Villuendas, and J. Tornos, "Single-mode optical-fiber sensors and tunable wavelength filters based on the resonant excitation of metal-clad modes," Appl. Opt. 33, 5197-5201 (1994).

20. J. Villatoro, D. Monzón-Hernández, and D. Talavera, "High resolution refractive index sensing with cladded multimode tapered optical fibre," Electron. Lett. 40, 106-107 (2004). 